International Journal of Pure and Applied Mathematics

Volume 100 No. 3 2015, 413-416

ISSN: 1311-8080 (printed version); ISSN: 1314-3395 (on-line version)

url: http://www.ijpam.eu

doi: http://dx.doi.org/10.12732/ijpam.v100i3.8

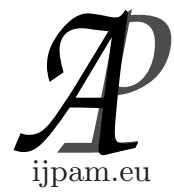

\title{
DYNAMICS OF THE DILATION WEIGHTED COMPOSITION OPERATORS
}

\author{
Bahmann Yousefi ${ }^{1}$, Fariba Ershad $^{2 \S}$ \\ ${ }^{1,2}$ Department of Mathematics \\ Payame Noor University \\ P.O. Box 19395-3697, Tehran, IRAN
}

\begin{abstract}
In this paper we investigate the hypercyclicity of adjoint of a special weighted composition operators on Hilbert spaces of analytic functions on the open unit disc.
\end{abstract}

AMS Subject Classification: 47B37, 47B38

Key Words: weighted composition operator, hypercyclicity, bounded point evaluation

\section{Introduction}

A bounded linear operator $T$ on a Hilbert space $H$ is said to be hypercyclic if there exists a vector $x \in H$ for which the orbit $\operatorname{Orb}(T, x)=\left\{T^{n} x: n \in \mathbb{N}\right\}$ is dense in $H$ and in this case we refer to $x$ as a hypercyclic vector for $T$. The holomorphic self maps of the open unit disk $U$ are divided into classes of elliptic and non-elliptic. The elliptic type is an automorphism and has a fixed point in $U$. It is well known that this map is conjugate to a rotation $z \rightarrow \lambda z$ for some complex number $\lambda$ with $|\lambda|=1$. The maps of that are not elliptic are called of non-elliptic type. The iterate of a non-elliptic map can be characterized by the Grand Iteration Theorem (see [6], p. 78). By $\psi^{\prime}(w)$ we denote the angular

Received: December 24, 2014

(C) 2015 Academic Publications, Ltd.

${ }^{\S}$ Correspondence author 
derivative of $\psi$ at $w \in \partial U$. Note that if $w \in U$, then $\psi^{\prime}(w)$ has the natural meaning of derivative. Also, by $\psi_{n}$ we mean the nth iteration of the function $\psi$.

Proposition 1.1. (see [6]) Suppose $\psi$ is a holomorphic self-map of $U$ that is not an elliptic automorphism. If $\psi$ has a fixed point $w \in U$, then $\psi_{n} \rightarrow w$ uniformly on compact subsets of $U$ and $\left|\psi^{\prime}(w)\right|<1$.

The unique attracting point $w$ in the above proposition is called the DenjoyWolff point of $\psi$, and $\psi$ is called a dilation of the open unit disk.

Definition 1.2. Let $H$ be a Hilbert space of analytic functions on the open unit disk $U$ and let $\psi$ be a dilation of the open unit disk $U$. We say that $C_{\psi}$ is quasi-contraction on $H$ if there exist a neighborhood $U_{w}$ of $w$ and $0<\alpha<1$ such that $\left|C_{\psi} f(z)\right| \leq \alpha|f(z)|$ for all $f, g \in H$, and every $z \in U_{w}$.

Let $H$ be a Hilbert space of analytic functions on the open unit disk $U$. For each $\lambda \in U$, the evaluation function $e_{\lambda}: H \rightarrow C$ is defined by $e_{\lambda}(f)=f(\lambda)$, $f \in H$. A complex valued function $\varphi$ on $U$ for which $\varphi H \subseteq H$ is called a multiplier of $H$. The set of all multipliers of $H$ is denoted by $M(H)$.

Consider the weighted composition operator $C_{\varphi, \psi}$ on a Hilbert space $H$ of analytic functions on the open unit disk $U$ defined by $C_{\varphi, \psi} f=\varphi$. $f \circ \psi(f \in H)$. We will investigate the hypercyclicity of the operator $C_{\varphi, \psi}^{*}$. For some sources we refer to [1]-[8].

\section{Main Results}

In this section $\psi$ will denote a holomorphic self-map of $U$ and $\varphi$ is a nonzero holomorphic map on $U$.

Note that if the adjoint of a continuous operator $T$ on a Banach space has an eigenvector, then $T^{*}$ fails to be hypercyclic (see [4]).

Lemma 2.1. Let $H$ be a separable Hilbert space of analytic functions on the open unit disk $U$ such that for each $\lambda \in U, e_{\lambda}$ is bounded on $H$. Also, let $\left\|M_{z}\right\| \leq 1$ on $H$, then $M(H)=H^{\infty}(U)$.

Proof. Let $f \in H^{\infty}(U)$. Then there is a sequence $\left\{p_{n}\right\}_{n}$ of polynomials converging to $f$ pointwise and for all $n,\left\|p_{n}\right\|_{U} \leq M$ for some $M>0$. Since $\left\|M_{z}\right\| \leq 1$ on $H$, by the Von-Neumann inequality, $\left\|M_{q}\right\|=\|q\|_{U}$ for all polynomials $q$. Hence we obtain $\left\|M_{p_{\mathrm{n}}}\right\| \leq M$ for all $n$. But ball $B(H)$ is compact 
in the weak operator topology and so by passing to a subsequence if necessary, we may assume that for some $A \in B(H), M_{p_{\mathrm{n}}} \longrightarrow A$ in the weak operator topology. Using the fact that $M_{p_{\mathrm{n}}}^{*} \longrightarrow A^{*}$ in the weak operator topology and acting these operators on $e_{\lambda}$ we get $p_{n}(\lambda) e_{\lambda}=M_{p_{\mathrm{n}}}^{*} e_{\lambda} \longrightarrow A^{*} e_{\lambda}$ weakly. Since $p_{n}(\lambda) \longrightarrow f(\lambda)$ we see that $A^{*} e_{\lambda}=f(\lambda) e_{\lambda}$ from which we can conclude that $A=M_{f}$ and this implies that $f \in M(H)$. Thus $H^{\infty}(U) \subset M(H)$. But it is well-known that $M(H) \subset H^{\infty}(U)$ (see [7]). Thus indeed, $M(H)=H^{\infty}(U)$.

Theorem 2.2. Let $H$ be a separable Hilbert space of analytic functions on the open unit disk $U$ such that $1 \in H$, for each $\lambda \in U, e_{\lambda}$ is bounded, and $\left\|M_{z}\right\| \leq 1$ on $H$. Furthermore, suppose that $\varphi \in M(H)$ and $\psi$ is a dilation map of $U$ with Denjoy-Wollf point $w$ such that $\varphi(w) \neq 0$, and let $C_{\psi}$ be a quasi-contraction. If $\left|\varphi \circ \psi_{n}(z)\right| \leq|\varphi(w)|$ eventually for all $n$ or $\|\psi\|_{U}<1$, then $\prod_{i=0}^{\infty} \frac{1}{\varphi(w)} \varphi \circ \psi_{i}$ is an eigenvector of $C_{\varphi, \psi}$ acting on $H$ and $C_{\varphi, \psi}^{*}$ is not hypercyclic.

Proof. First, let $\left|\varphi \circ \psi_{n}(z)\right| \leq|\varphi(w)|$ eventually for all $n$. Since $C_{\psi}$ is quasi-contraction, there exist a neighborhood $U_{w}$ of $w$ and $0<\alpha<1$ such that

$$
\left|C_{\psi} f(z)-C_{\psi} g(z)\right| \leq \alpha|f(z)-g(z)|
$$

for all $f, g \in H$, and every $z \in U_{w}$. Let $n>2$ and define $f=\varphi \circ \psi_{n-1}$ and $g=\varphi(w)$. Note that $\varphi \circ \psi_{n}=C_{\psi} \varphi \circ \psi_{n-1}$, and since $\psi_{n}(z) \rightarrow w$, for $n$ large enough we have $\psi_{n-1} \in U_{w}$. Clearly, $f$ and $g$ are in $H$ and by using (*) we have

$$
\begin{aligned}
\left|\varphi \circ \psi_{n}(z)-\varphi(w)\right| & \leq \alpha\left|\varphi \circ \psi_{n-1}(z)-\varphi(w)\right| \\
& \leq \alpha^{n}|\varphi(z)-\varphi(w)|
\end{aligned}
$$

for all $z \in U_{w}$ and $n$ large enough. Therefore,

$$
\left|1-\frac{1}{\varphi(w)} \varphi\left(\psi_{n}(z)\right)\right| \leq\left(1+\frac{1}{|\varphi(w)|}|\varphi(z)|\right) \alpha^{n} .
$$

Thus $\sum_{n=0}^{\infty}\left|1-\frac{1}{\varphi(w)} \varphi\left(\psi_{n}(z)\right)\right|$ converges uniformly on $K$. Hence $\prod_{n=0}^{\infty} \frac{1}{\varphi(w)} \varphi\left(\psi_{n}(z)\right)$ also converges uniformly on K. Define $g(z)=\prod_{n=0}^{\infty} \frac{1}{\varphi(w)} \varphi\left(\psi_{n}(z)\right)$. Thus $g$ is nonzero holomorphic function on $U$ and by our assumption, $g \in H^{\infty}(U)$. Now 
by the Lemma 2.1, $g \in M(H)$. But $1 \in H$, thus indeed $g \in H$. Note that $\varphi(z) \cdot g(\psi(z))=\varphi(w) g(z)$, hence $\prod_{i=0}^{\infty} \frac{1}{\varphi(w)} \varphi \circ \psi_{i}$ is an eigenvector of $C_{\varphi, \psi}$. Now let $\|\psi\|_{U}<1$ and note that $C_{\varphi, \psi}^{n} g=\varphi(w)^{n} g$. Thus

$$
g=\varphi(w)^{-n} \prod_{j=0}^{n-1} \varphi\left(\psi_{j}\right) g \circ \psi_{n}
$$

Since $\|\psi\|_{U}<1$, clearly $g \in H^{\infty}(U)$ and so $g \in H$. Also, $\prod_{i=0}^{\infty} \frac{1}{\varphi(w)} \varphi \circ \psi_{i}$ is an eigenvector of $C_{\varphi, \psi}$. Since in each case $\varphi(w)$ is an eigenvalue of $C_{\varphi, \psi}$, hence $C_{\varphi, \psi}^{*}$ is not hypercyclic and so the proof is complete.

\section{References}

[1] P. S. Bourdon and J. H. Shapiro, Cyclic composition operators on $H^{2}$, Proc. Symp. Pure Math., 51, No. 2 (1990), 43-53.

[2] P. S. Bourdon and J. H. Shapiro, Cyclic phenomena for composition operators , Mem. Amer. Math. Soc., 596, No. 125 (1997), 1-150.

[3] T. Gamelin, Uniform Algebra, New York (1984).

[4] G. Godefroy and J. H. Shapiro, Operators with dense invariant cyclic vector manifolds, J. Func. Anal., 98 (1991), 229-269.

[5] J. E. Marsden and M. J. Hoffman, Basic Complex Analysis, 2nd ed., W. H. Freeman and Company, New York (1987).

[6] J. H. Shapiro, Composition Operators and Classical Function Theory, Springer-Verlag New York, (1993).

[7] A. Shields and L. Wallen, The commutant of certain Hilbert space operators, Indiana Univ. Math. J., 20 (1971), 777-788.

[8] B. Yousefi and H. Rezaei, Hypercyclic property of weighted composition operators, Proc. Amer. Math. Soc., 135, No. 10 (2007), 3263-3271. 